\title{
Object-oriented codes representation of program understanding system
}

\begin{abstract}
The purpose of this paper is to present and describe the representation of an object-oriented programming code in the program understanding system. The programôs representation that has been used and will be discussed is an Object-oriented Control Flow Graph (OO-CFG). OO-CFG was an added version on the notation of the traditional control flow graph (CFG). This new OO-CFG has been created since from the literature there are no researches specifically focus on the problem in presenting the communications between objects. Usually the communication between objects in object-oriented program code is by a process of message passing. In this paper, we are introducing the OO-CFG representation and also example on the usage.
\end{abstract}

Keyword: Object-oriented program code; Program understanding system; Object-oriented Control Flow Graph (OO-CFG) 